\title{
Morfología del polen en especies de Canna (Cannaceae) y su implicancia sistemática
}

\author{
María de las Mercedes Ciciarelli, Lilian M. Passarelli \& Cristina H. Rolleri \\ Laboratorio de Estudios de Anatomía Vegetal Evolutiva y Sistemática (LEAVES), Facultad de Ciencias Naturales y \\ Museo de La Plata, 64 entre 120 y diagonal 113, B1904 DZB, La Plata, Argentina Tel. +4249049; \\ mmciciar@yahoo.com.ar, lmpassarelli@yahoo.com.ar, tinar@speedy.com.ar
}

\author{
Recibido 17-IV-2009. Corregido 20-VIII-2009. Aceptado 21-IX-2009.
}

\begin{abstract}
Pollen morphology in species of Canna (Cannaceae), and systematics implications. The morphology of pollen grains of eight taxa of Canna, C. ascendens, C. coccinea, C. compacta, C. glauca, C. indica, C. paniculata, $C$. variegatifolia and C. fuchsina, an unpublished new species, were studied using light and scanning electronic microscopes. We used the Wodehouse technique on samples of 20 grains per specimen to measure the intine with a light microscope; and the density of spines (in $400 \mu \mathrm{m}^{2}$ fields) with scanning electronic microscopy. Pollen grains are spherical, echinate, omniaperturate. The sporoderm presents a very thin exine covering a thicker intine. Corrugate micro-perforate, sub-reticulate, rugate, rugulate, striate to folded, micro-striate, micro-granulate, and smooth types of the external surface of the sporoderm were found. The spines consist of exine, partially to completely covered by tryphine. The two-layered intine is the thicker part of the wall. Echinate ornamentation is a generic character in Canna, but size, surface and color of pollen walls, and density and shape of spines, are diagnostic for species. Pollen morphology supports the view of $C$. indica and $C$. coccinea as different species. Canna fuchsina grows in wild, dense colonies, in humid riverside forests from Buenos Aires and Santa Fe Provinces, Argentina; its characters suggest relationships with a not well known group of taxa, some of them hybrids, such as C. $x$ generalis. However, as these plants showed normal, well formed grains, close to those of $C$. coccinea, that germinate over the stigmatic surfaces in fresh flowers, we decided to include their pollen in this study. Rev. Biol. Trop. 58 (1): 63-79. Epub 2010 March 01.
\end{abstract}

Key words: Canna, Cannaceae, pollen, omniaperturate grains, echinate ornamentation, spines, exine, intine, tryphine, systematics, palynology.

Las Cannaceae Link. son originarias de América y habitan con preferencia en los trópicos y subtrópicos del Nuevo Mundo. Sus inflorescencias y flores llamativas e intensamente coloreadas han llevado al cultivo de las plantas como ornamentales y a la producción de numerosos híbridos de jardinería. Han sido introducidas en Europa, Micronesia, Melanesia, Polinesia y el Sur de China y se naturalizan con facilidad (Tutin et al. 1980, Tanaka 2001).

Juntamente con otras siete familias, Costaceae (K. Schum.) Nak., Heliconiaceae (Endl.) Nak., Lowiaceae Ridley, Marantaceae Petersen, Strelitiziaceae (K. Schum.) Hutch., Musaceae
A. L. de Jussieu y Zingiberaceae Adanson, están incluidas en el orden Zingiberales, considerado monofilético por varios autores (Tomlinson 1962, 1969, Dahlgren \& Rassmusen 1983, Kress 1990, 1995, Chase et al. 1993, Smith et al. 1993, Stevenson \& Loconte 1995, Kress et al. 2001). De acuerdo con ese enfoque, Cannaceae, Costaceae, Marantaceae y Zingiberaceae formarían un clado único basado en la reducción del androceo a un solo estambre funcional y los estaminodios grandes y llamativos, entre otros caracteres (Judd et al. 2002).

El número de especies asignado al género ha variado con el conocimiento de éste y aún 
es objeto de cierta discrepancia. Horaninow (1862) menciona 100 especies; Bouché (1833) y Bouché (1844) reporta 47 y 71, respectivamente; Kranzlin (1912) y Winkler (1930), 50; Kress \& Prince (2000) toman en consideración 3 sólo para Estados Unidos de América; Delin \& Kress (2000) sugieren la existencia de entre 10 y 20 especies silvestres y Tanaka (2001) reconoce 19, 15 de las cuales también se encuentran en Asia. Este autor cita 4 especies para Argentina, pero no trata en su revisión $C$. variegatifolia Ciciarelli (Ciciarelli 1995) ni la más reciente, $C$. ascendens Ciciarelli (Ciciarelli 2007), lo que elevaría el número de especies a 21, de las cuales, 15 crecen silvestres en Argentina (Ciciarelli 1989, 1995, 2007, Ciciarelli \& Rolleri 2008). La obra más reciente de MaasVan de Kamer \& Maas (2008) vuelve a proponer la existencia de sólo 10 especies silvestres en el mundo.

Los estudios palinológicos en relación con la sistemática de Canna son escasos y remiten a Tanaka (2001), Ciciarelli (2007) y Ciciarelli \& Rolleri (2008). Tanaka (2001) ilustró el polen de $C$. indica L., C. jacobiniflora T. Koyama $\&$ Nb. Tanaka y $C$. discolor Lindl. Ciciarelli (2007) analizó comparativamente los granos de $C$. ascendens y $C$. variegatifolia. Ciciarelli \& Rolleri (2008) describieron brevemente el polen de siete especies neotropicales e ilustraron los granos de $C$. ascendens, $C$. indica y $C$. variegatifolia, encontraron cierta uniformidad morfológica en el polen del género y destacaron que algunos rasgos podrían ser diagnósticos si se profundizara el estudio de las paredes y las espinas de las especies.

El estudio de la estructura del esporodermo de los granos de polen de especies de Canna, su composición química y germinación, estuvo particularmente centrado, con pocas excepciones, en $C$. indica, con aportes de Wodehouse (1932, 1935), Erdtman (1960 b), Nair (1960), Skvarla \& Rowley (1970, 1974, 1975), Segeren \& Maas (1971) y Kress \& Stone (1982). Estos últimos consideraron que el esporodermo de $C$. indica estaba formado por una delgada capa de exina, presente también en las espinas y una doble capa de intina; describieron una exintina con canales y una endintina delgada y fibrosa, adyacente al protoplasto. Skvarla \& Rowley (1970) estudiaron C. generalis y describieron un esporodermo grueso con exina delgada, incompleta, bien representada en las espinas pero a veces ausente en la superficie entre espinas, formada por esporopolenina. En granos en desarrollo determinaron la presencia de esporopolenina en la intina, en forma de inclusiones en la endintina, cerca de la membrana plasmática. También detectaron trifina, en depósitos dispersos, en exintina y espinas. Describieron una exina delgada y consideraron que las espinas serían parte de la exina.

Estudios recientes del género (Ciciarelli 2007, Ciciarelli \& Rolleri 2008, Ciciarelli et al. 2009), sugieren que la morfología del polen de las especies aportaría nuevos datos importantes de valor sistemático. Por eso, en este trabajo se consideró conveniente estudiar detalladamente los caracteres palinológicos de ocho especies seleccionadas: $C$. ascendens, $C$. coccinea, C. compacta, C. glauca, C. indica, C. paniculata, C. variegatifolia y C. fuchsina sp. ined. Un estudio fenológico y morfológico efectuado en colonias silvestres de C. fuchsina, durante cinco años, indicó que tiene afinidades con $C$. flaccida Salisbury y con los táxones que se agruparon sub C. $x$ generalis Bailey, originalmente descritos por Bailey (1923) como híbridos. Las plantas tienen, invariablemente, flores de color rosa intenso a fucsia, estaminodios anchos, producen polen normal, semillas abundantes y se reproducen normalmente por vía sexual. La morfología del polen permitió completar el análisis que llevó a tratarla como una nueva especie (Ciciarelli et al. 2009).

\section{MATERIALES Y MÉTODOS}

Se trabajó con material fresco que se comparó con ejemplares herborizados del Herbario de la Facultad de Ciencias Naturales y Museo de La Plata (LP), del Herbario del InstitutoFundación Miguel Lillo, Tucumán (LIL) y Herbario del Instituto de Botánica Darwinion (SI).

Los granos de polen se observaron con microscopio de luz (en adelante ML) y 
electrónico de barrido (en adelante MEB). Se trataron con la técnica de Woodehouse (1935) porque ni los granos frescos ni los provenientes de material herborizado resisten la técnica de acetólisis tradicional de Erdtman (1960 a), algo ya observado por Skvarla y Rowley (1970).

Para observaciones con ML y para analizar la composición química del esporodermo, los granos frescos se colorearon con azul de toluidina (TBO) $1 \%$ en agua y con fucsina básica. Se montaron con glicerina-gelatina y se sellaron con parafina. Para la observación con MEB, los granos de polen se colocaron sobre cinta adhesiva doble faz, fueron metalizados con oro paladio y examinados con un microscopio JEOL JSM T-1100, en el Servicio de Microscopía Electrónica de la Facultad de Ciencias Naturales y Museo de La Plata.

Las medidas de los granos de polen y el espesor de la pared se basan en 20 mediciones por ejemplar y se efectuaron con ML. Sobre las fotos obtenidas con MEB se midió la densidad de las espinas, en campos de $400 \mu \mathrm{m}^{2}$.

En la escala de valores tonales, en la que los extremos son el blanco y el negro, existe una amplia gama de grises intermedios. Con MEB, la pared y las espinas pueden presentar distintos valores de la intensidad luminosa del tono. Valor tonal o simplemente valor, es el término aplicado para describir estas diferencias.

La terminología palinológica utilizada es la clásica para este tipo de estudios; el término omniaberturado se aplica según Skvarla \& Rowley (1970) y Punt et al. (2007).

\section{RESULTADOS}

Los granos de polen son esferoidales y omniaberturados, es decir, sin una región abertural visible y con una considerable reducción de la exina, carácter propio de las aberturas, por lo que se considera que la superficie completa del grano es de naturaleza abertural. Tienen tamaños que van desde 55 hasta $90 \mu \mathrm{m}$ (Cuadro 1). Las muestras analizadas mostraron granos de aspecto normal en casi todos los táxones analizados, excepto en $C$. coccinea, en la que se observaron granos de diferentes tamaños, algunos comparativamente muy pequeños o colapsados (Fig. 1E-F). Sin embargo, los recuentos efectuados en distintos especímenes de esta especie indicaron que la proporción de granos de aspecto normal llega al 50\%. En los demás táxones se hallaron proporciones del $95 \%$ o más de granos normales, y escasos granos irregulares, generalmente de menor tamaño (Fig. 1A-D).

La ornamentación del esporodermo es equinada. Las espinas pueden ser típicamente cónicas, irregulares a digitiformes o gemiformes, o bien semejantes a verrugas mamiformes.

Se encontraron espinas cónicas típicas, con ápices agudos en C. ascendens (Fig. 2E, 6C), C. indica (Fig. 2A-B, 4D, 6B) y C. variegatifolia (Fig. 2C-D, 4B, 6D), con ápices redondeados en C. glauca (Fig. 5A-B, 6E), y con ápices truncados o torcidos en C. paniculata (Fig. 1D, 2F, 5D, 6A).

Las espinas romas, como verrugas mamiformes, se encontraron en $C$. coccinea (Fig. 1E-F, 3A, 6H-I) y C. fuchsina (Fig. 3B-D, 5C, $6 \mathrm{G})$. Finalmente, se hallaron espinas digitiformes a irregulares, a veces gemiformes, en $C$. compacta (Fig. 5E-F, 6F).

La relación entre el ancho basal y la altura de estos procesos varía: en $C$. ascendens, $C$. compacta y $C$. variegatifolia, el alto es igual al ancho basal; en $C$. coccinea, $C$. fuchsina y $C$. paniculata, las espinas tienen bases cuyo diámetro es mayor que su altura mientras que en C. glauca y $C$. indica esta relación es inversa, es decir, las espinas son más altas que anchas en la base. Las espinas más largas se registraron en $C$. indica y las más cortas en $C$. variegatifolia. Además de las variaciones en longitud y anchura de sus bases, las espinas difieren en la densidad de su distribución. Las medidas y densidades de estos procesos en cada especie se consignan en el Cuadro 1.

La pared de los granos presenta una exina muy delgada y una intina más gruesa.

Sobre la exina se encuentra con frecuencia trifina, una sustancia procedente de la desintegración del tapete y que contiene materiales de las membranas rotas de las organelas celulares de aquél. La trifina no se encuentra 


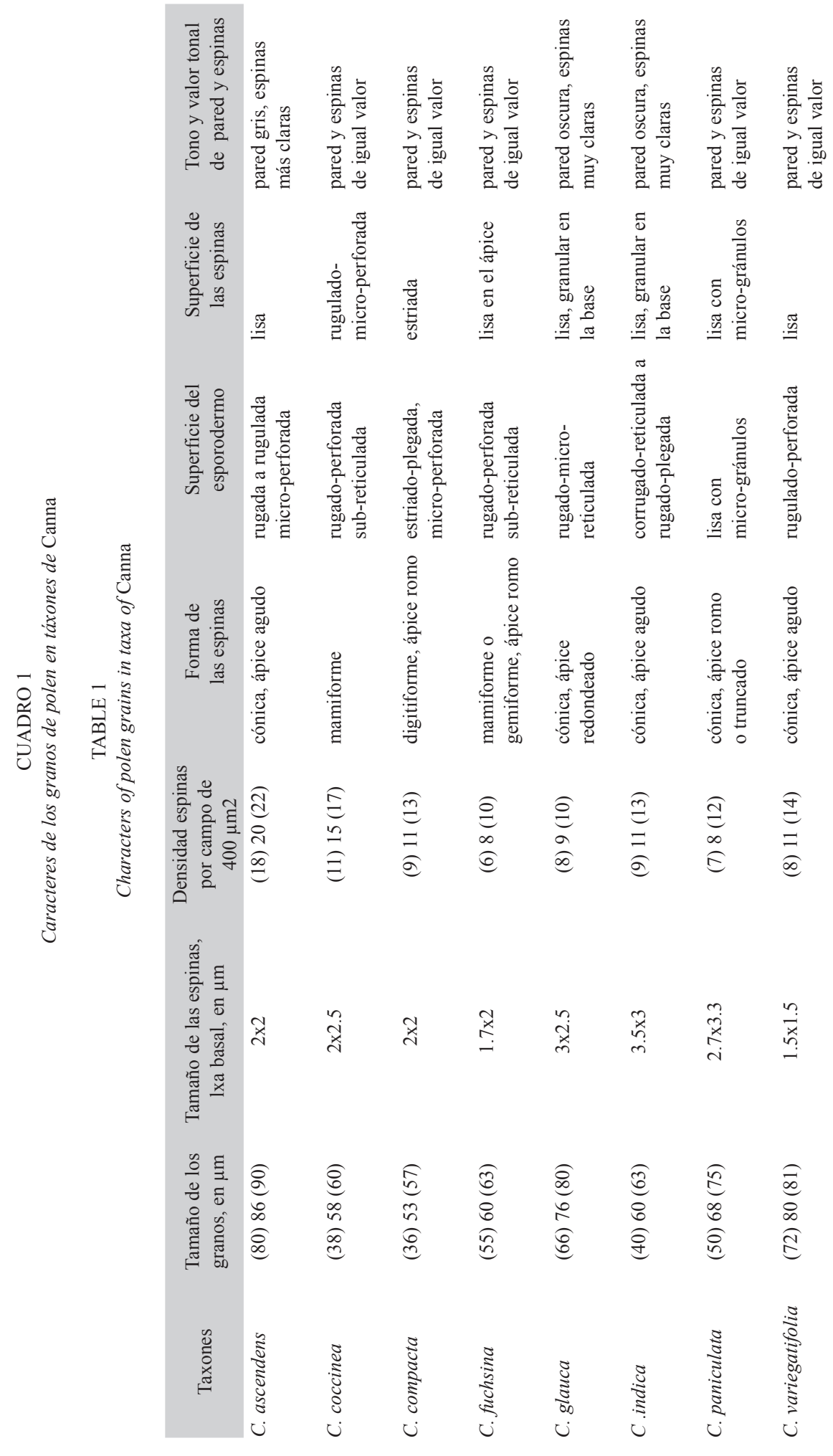


en forma de una capa completa sino en manchas o pátinas esporádicas de extensión variable (Fig. 2D, 5A).

La exina, formada por esporopolenina, es muy delgada. Su espesor no se distingue con ML, solamente mediante la observación con MEB, en zonas rasgadas de la pared se puede apreciarla y además, visualizar las variaciones que caracterizan el depósito de esta capa, no siempre completa o uniformemente distribuida sobre la intina. No se detectan capas, excepto una ectexina delicada, que en algunos casos se rompe con facilidad en fisuras que dejan ver la exintina oscura, como sucede espontáneamente en granos de polen de C. indica (Fig. 2B); en esta especie también se observa cuando se produce la ruptura accidental de las espinas (Fig. 2A).

Las espinas son parte de la ectexina y también están constituidas por esporopolenina. En granos de algunas especies la micrornamentación se interrumpe en la base de las espinas o cubre algo menos de la mitad basal de éstas, en forma de una zona densamente microgranular, como en C. ascendens (Fig. 4A, 6C), C. glauca (Fig. 5A-B, 6E), C. indica (Fig. 4D, 6B), $C$. paniculata (Fig. 5C, 6A) y C. variegatifolia (Fig. 4B, 6D); mientras que en $C$. compacta (Fig. 5E-F, 6F), C. coccinea (Fig. 4C, 6H-I) y C. fuchsina (Fig. 5D, 6G) cubre totalmente o casi totalmente estos procesos. Las espinas no cubiertas por la exina suelen tener tono blanco, brillante, y se destacan del resto de la pared, que es gris más oscuro, algo que puede ser más notable en granos de $C$. glauca, $C$. indica y C. variegatifolia. Las espinas mamiformes, gemiformes o digitiformes de los granos de C. coccinea, C. compacta y C. fuchsina, totalmente o casi totalmente cubiertas por exina, tienen el mismo valor del tono que la pared circundante.

La superficie de la pared es variable en las especies. Es corrugado-reticulada a rugadoplegada en $C$. indica (Fig. 4D), rugado-microrreticulada en $C$. glauca (Fig. 5A-B), rugada a rugulada, microperforada en $C$. ascendens (Fig. 4A), rugulado-perforada en C. variegatifolia (Fig. 4B), rugado-perforada, sub-reticulada en
C. coccinea (Fig. 4C) y C. fuchsina (Fig. 5D), estriado-plegada, microperforada, en $C$. compacta (Fig. 5E-F) y finalmente, lisa con microgránulos, en $C$. paniculata (Fig. 5C).

La intina puede tener desde 4 hasta $10 \mu \mathrm{m}$ de espesor, según los táxones. Representa el mayor espesor de la pared y su grosor varía en granos de una misma especie, según el grado de madurez de éstos: en muestras de granos juveniles tomadas antes de la antesis, el espesor de la intina es mayor que en granos maduros, provenientes de muestras tomadas en la superficie del estilo. Está formada por dos capas, la endintina y la exintina, visibles al ML.

Las respuestas a las pruebas microquímicas son diferentes en la pared de los granos y en el citoplasma de éstos. Ante el TBO, el citoplasma da color rosa púrpura por la presencia de polisacáridos, la intina no se colorea en tanto que en la parte más externa de la pared, una delgada capa sobre la exina, da color verde intenso, lo que se atribuye a la presencia de sustancias relacionadas con la secreción del tapete, como polifosfatos y trifina. Ésta aparece sobre la superficie de la exina en forma de pátinas irregulares, no siempre de manera uniforme ni completa, tapando ocasionalmente grupos amplios de espinas. Ante la fucsina básica, coloración específica para esporopolenina, reaccionan de manera similar la exina y las espinas.

En tres táxones, C. fuchsina (Fig. 3B-D), C. indica (Fig. 2B) y C. variegatifolia (Fig. 3D), los granos se han observado, in situ e in vitro, en el proceso de emisión de los tubos polínicos.

\section{DESCRIPCIÓN PORMENORIZADA DE LAS ESPECIES}

\section{Canna ascendens Ciciarelli}

(Fig. 1A, 2E, 4A, 6C)

Granos de polen esferoidales, de (80) 86 (90) $\mu \mathrm{m}$, omniaberturados, equinados. Espinas cónicas, con ápices agudos, de $2 \times 2 \mu \mathrm{m}$, de tono gris claro, como el resto del esporodermo; densidad 18-22 espinas/campo de 

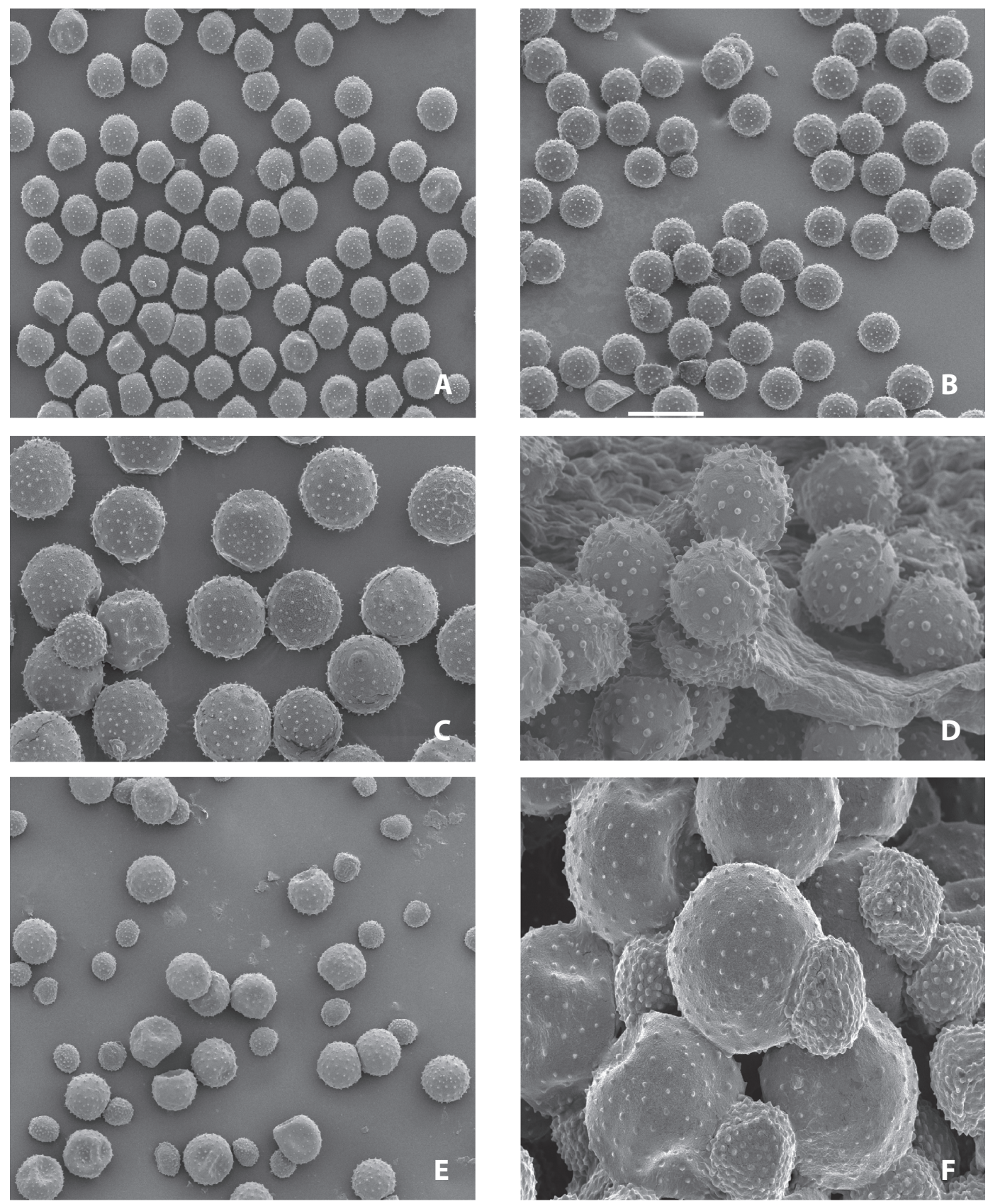

Fig.1. Granos de polen de especies de Canna: vistas panorámicas. A: C. ascendens. B: C. variegatifolia. C: C. indica. D: C. paniculata. E-F: C. coccinea, granos de diferentes tamaños. Barra $=100 \mu \mathrm{m}$ en A, B, E; $50 \mu \mathrm{m}$ en C; $40 \mu \mathrm{m}$ en D; 25 $\mu \mathrm{m}$ en $\mathrm{F}$.

Fig.1. Pollen grains in species of Canna, panoramic views. A: C. ascendens. B: C. variegatifolia. C: C. indica. D: C. paniculata. E-F: C. coccinea, grains of different sizes. Bar $=100 \mu \mathrm{m}$ in A, B, E; $50 \mu \mathrm{m}$ in C; $40 \mu \mathrm{m}$ in D; $25 \mu \mathrm{m}$ in F. 
$400 \mu \mathrm{m}^{2}$. Superficie de la exina rugada a rugulada microperforada; micrornamentación de la exina extendida hasta la mitad de la altura de las espinas. Superficie de las espinas lisa.

Ejemplares de referencia seleccionados

ARGENTINA: Buenos Aires: Ensenada, España y Dr. Garay, en canal, Ciciarelli et al. 16 (LP). Camino a Isla Santiago, cerca de Bossinga, Ciciarelli et al. 17 (LP). La Plata, Ringuelet, baldíos en alrededores de calles 511 y 22, Ciciarelli 10 (LP). City Bell, cruce de Alvear con Sarmiento, Ciciarelli 9 (LP).

\section{Canna coccinea Miller}

(Fig. 1E-F, 3A, 4C, 6H-I)

Granos de polen esferoidales, de (38) 58 (60) $\mu \mathrm{m}$, omniaberturados, verrugosos a subequinados. Espinas mamiformes, de $2 \times 2.5 \mu \mathrm{m}$, de tono gris claro, como el resto del esporodermo, cubiertas por la exina, con base muy ensanchada y ápice romo o truncado; densidad 11-17 espinas/campo de $400 \mu \mathrm{m}^{2}$. Exina rugado-perforada, sub-reticulada. Superficie de los procesos o espinas rugulado-microperforada.

\section{Ejemplares de referencia seleccionados}

ARGENTINA: Buenos Aires: Ensenada, camino a Isla Santiago, por Bossinga, Ciciarelli et. al. 18 ( LP). La Plata, Punta Lara, Fabris 7517 (LP). Delta, El Tigre, Hicken 26940 (SI). Delta, Paraná de Las Palmas, Burkart 9260 (SI). Capital, Palermo, Hicken 26941 (SI). Chaco: Schulz \& Benitez 26 (LP). Córdoba: Colón, Quebrada de Ascochinga, Girardelli 1194 (SI). Formosa: Monte Lasten, Jörgensen 2303 (LIL). Misiones: Candelaria, Santa Ana, Rodríguez 672 (SI).Tucumán: Capital, Empalme Centenario, Colmenar, Villa 131568 (LIL). Capital, entre Capital y Muñecas, Meyer 13592 (LIL). Muñecas, Schreiber 51603 (LIL). Muñecas, s/c 331 (LIL).

\section{Canna compacta Roscoe}

(Fig. 5E-F, 6F)

Granos de polen esferoidales, de (36) 53 (57) $\mu \mathrm{m}$, omniaberturados, equinados. Espinas digitiformes, con ápice romo, de $2 \times 2 \mu \mathrm{m}$, de tono gris claro, como el resto del esporodermo; densidad 9-13 espinas/campo de $400 \mu \mathrm{m}^{2}$. Exina estriado-plegada, micro-perforada. Superficie de los procesos o espinas estriada.

\section{Ejemplares de referencia seleccionados}

ARGENTINA: Jujuy: Yala, Fabris 8547 (LP). Yala, Cabrera y Fabris 20055 (LP) y Cabrera et al. 24000 (LP). Quebrada de Yala, Cabrera y Fabris 19964 (LP). Salta: San Ramón de la Nueva Orán, Finca de Yakúlikas, al SE de las Serranías de Las Pavas, cerca de la localidad Aguas Blancas, Palací 3 (LP). Anta, Parque Nacional El Rey, Chalukian 7 (LP); Rosario de Lerma, Santa Rosa de Tastil, Fabris 8548 y 8549 (LP). Tucumán: Capital, Río Sali, s. coll. 19004 (LP). Capital, Meyer 3381 (LIL). Chicligasta, Puesto Las Pavas, Meyer 1802 (LP). Puesto Santa Rosa, Meyer 15468 (LIL). Dique Pueblo Chico, Escaba, Monetti 1591 (LIL). Tafí, entre Tafí Viejo y Los Nogales, ruta 9, Meyer y Vaca Gómez 22313 (LIL).

BRASIL: Amazonas: Itapiranga, Smith y Reitz 12640 (SI).

\section{Canna fuchsina Ciciarelli}

(Fig. 3B-D, 5C, 6I)

Granos de polen esferoidales, de (55) 60 (63) $\mu \mathrm{m}$, omniaberturados, verrugosos a subequinados. Procesos mamiformes o gemiformes, con ápice romo, más anchos que altos, de 1.7x $2.2 \mu \mathrm{m}$; densidad 6-10 procesos/campo de $400 \mu \mathrm{m}^{2}$. Exina de tono gris claro, no extendida totalmente sobre los procesos, con la superficie rugado-perforada, sub-reticulada. Superficie de 

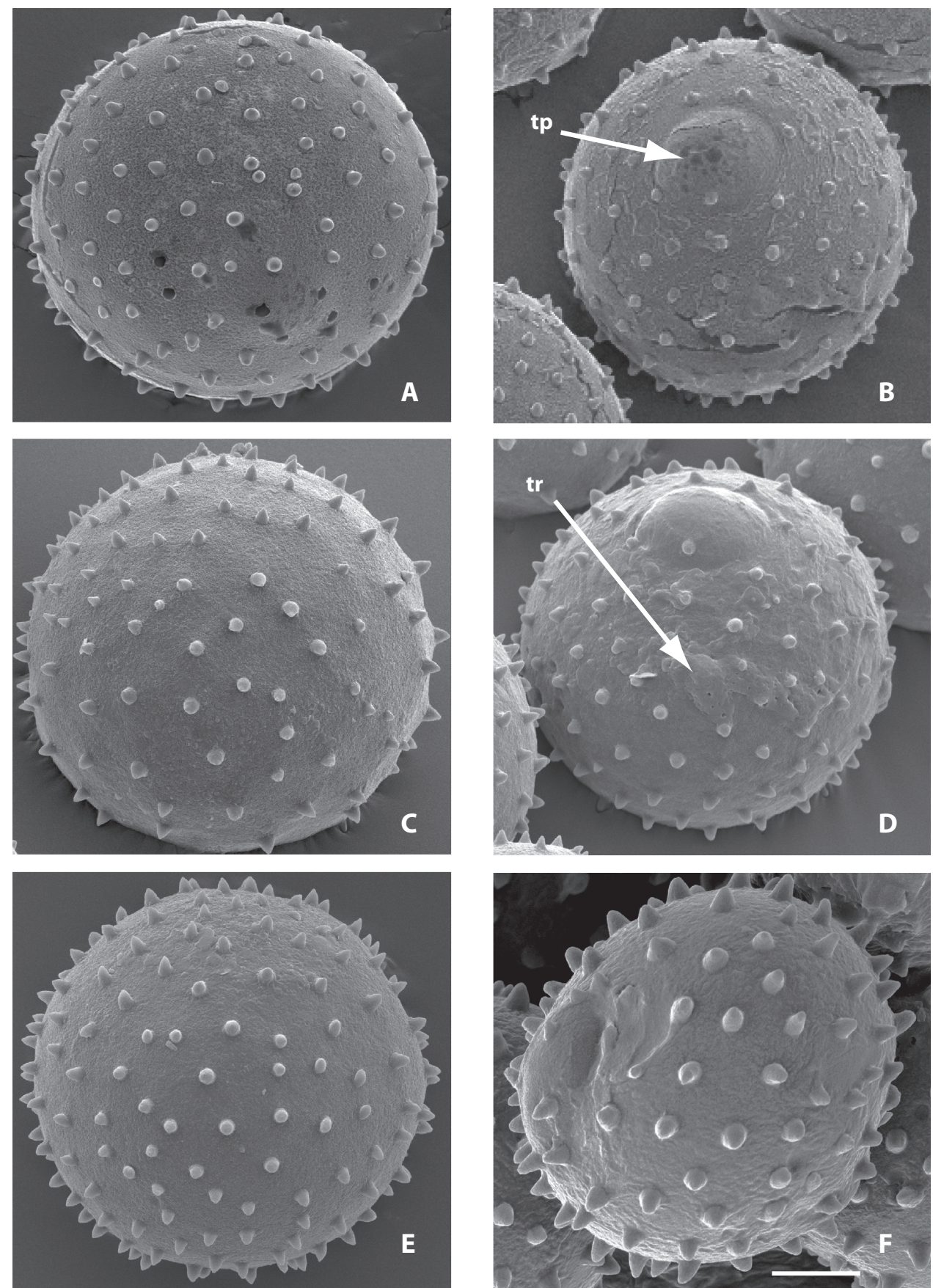

Fig. 2. Granos de polen en especies de Canna. A-B: C. indica. A: Grano adulto. B: Grano en proceso de germinación. C-D: C. variegatifolia. C: Grano adulto. D: Grano en proceso de germinación. E: C. ascendens, grano adulto. F: C. paniculata, grano adulto. tp, emergencia de tubo polínico; tr, trifina. Bar $=14 \mu \mathrm{m}$ en A-B, D; $15 \mu \mathrm{m}$ en C; $19 \mu \mathrm{m}$ en E; 16 en F.

Fig. 2. Pollen grains in species of Canna. A-B: C. indica. A: Adult grain. B: Adult grain beginning to emite pollen tube. C-D: C. variegatifolia. C: Adult grain. D: Adult grain beginning to emite pollen tube. E: C. ascendens, adult grain. F: C. paniculata, adult grain. tp, pollen tube; tr, tryphine. $\mathrm{Bar}=14 \mu \mathrm{m}$ in $\mathrm{A}-\mathrm{B}, \mathrm{D} ; 15 \mu \mathrm{m}$ in $\mathrm{C} ; 19 \mu \mathrm{m}$ en $\mathrm{E}$; 16 in $\mathrm{F}$. 

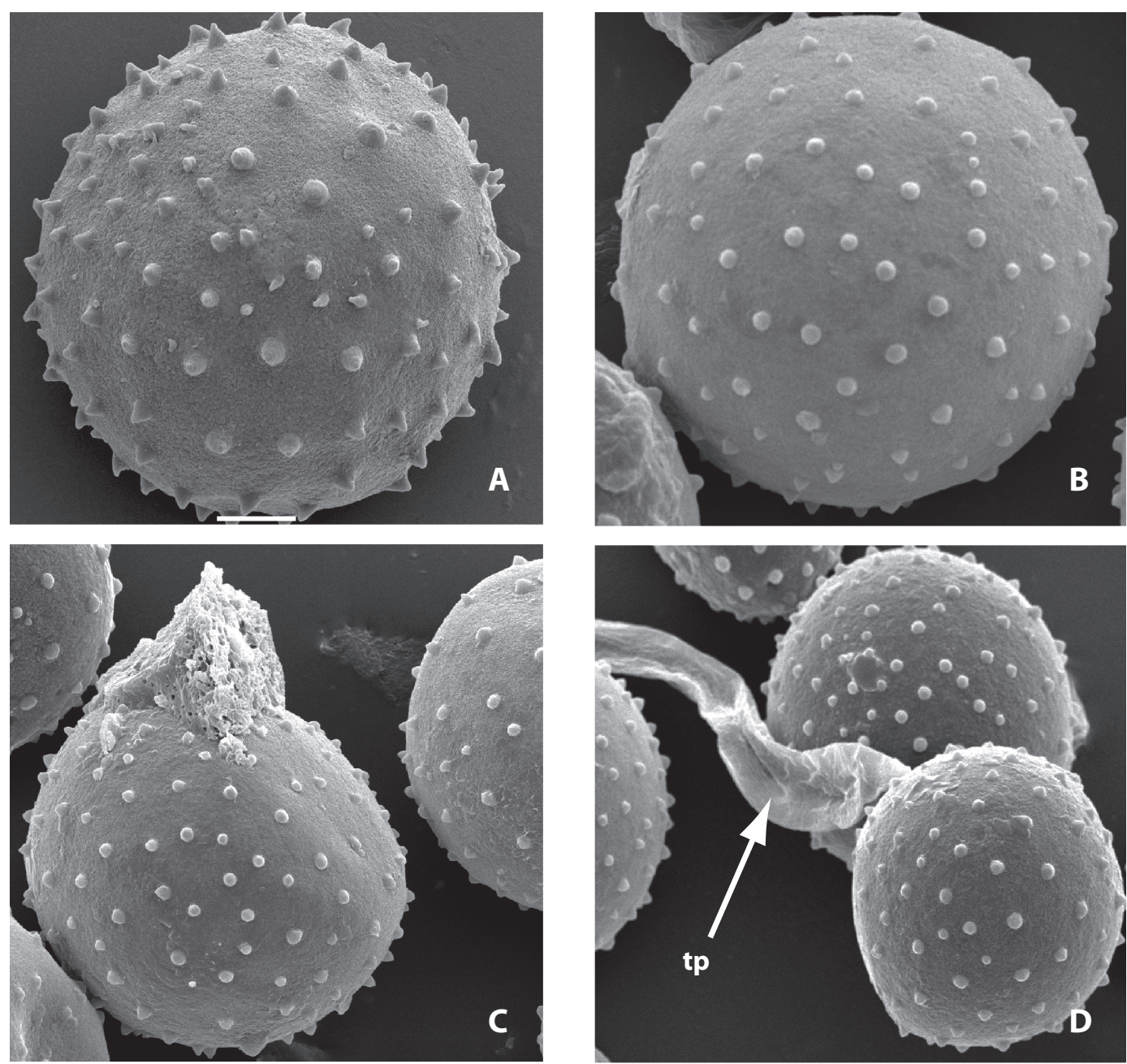

Fig. 3. Granos de polen de especies de Canna. A: C. coccinea, grano adulto. B-D: Canna sp. B: Grano adulto. C: Grano adulto en el inicio de la emisión del tubo polínico. D: Tubo polínico en desarrollo. tp, tubo polínico; tr, trifina. Barra= 10 $\mu \mathrm{m}$ en $\mathrm{A}-\mathrm{B} ; 17 \mu \mathrm{m}$ en $\mathrm{C}-\mathrm{D}$.

Fig. 3. Pollen grains in species of Canna. A: C. coccinea, adult grain. B-D: Canna sp. B: Adult grain. C: Adult grain at the beginning of emergence of pollen tube. D: Developing pollen tube. tp, pollen tube; tr, tryphine. Bar= $10 \mu \mathrm{m}$ in A-B; $17 \mu \mathrm{m}$ in C-D.

los procesos rugado-perforada, sub-reticulada, lisa en el ápice.

\section{Ejemplares de referencia seleccionados}

ARGENTINA: Buenos Aires: City Bell, Pellegrini, en baldíos cerca de calles 5 y 6 , Ciciarelli 11 (LP). Santa Fe: Providencia, al borde de los caminos, Ciciarelli 15 (LP).

\section{Canna glauca $\mathrm{L}$.}

(Fig. 5A-B, 6E)

Granos de polen esferoidales, de (66) 76 (80) $\mu \mathrm{m}$, omniaberturados, equinados. Espinas cónicas, con ápice redondeado, más altas que anchas, $3 \times 2.5 \mu \mathrm{m}$; densidad $8-10$ espinas/ campo de $400 \mu \mathrm{m}^{2}$. La exina se extiende hasta 

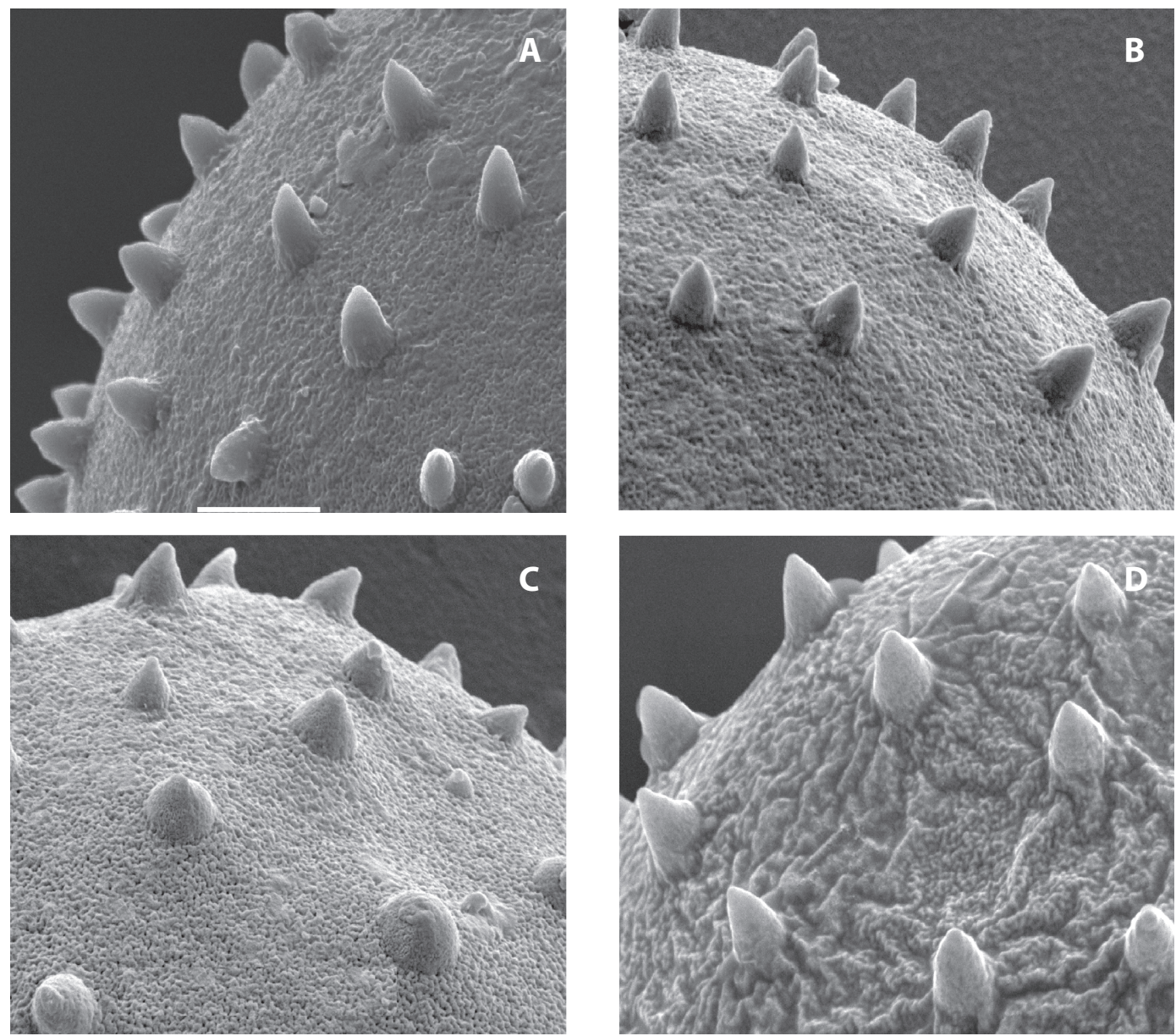

Fig. 4. Ornamentación en granos de polen de especies de Canna. A: C. ascendens. B: C. variegatifolia. C: C. coccinea. D: C. indica. Barra $=3 \mu \mathrm{m}$ en A; $4 \mu \mathrm{m}$ en B-C; $7 \mu \mathrm{m}$ en D

Fig. 4. Ornamentation of pollen grains in species of Canna. A: C. ascendens. B: C. variegatifolia. C: C. coccinea. D: C. indica. $\mathrm{Bar}=3 \mu \mathrm{m}$ in $\mathrm{A} ; 4 \mu \mathrm{m}$ in $\mathrm{B}-\mathrm{C} ; 7 \mu \mathrm{m}$ in $\mathrm{D}$.

la base de las mismas. Exina gris oscuro, espinas muy claras y brillantes. Superficie de la exina rugada, micro-reticulada. Superficie de las espinas lisa, granular en la base.

Ejemplares de referencia seleccionados

ARGENTINA: Buenos Aires: Ensenada, Punta Lara, Cabrera 4897, 617 (ambos LP). La Balandra, Ciciarelli et. al. 19 (LP). Capital, Palermo, Hicken 26937 (SI). Barracas al Sur, Venturi 52 (SI). El Tigre, Lanfranchi 506 (SI). Isla Paulino, Marelli 1905 (SI). Ensenada,
Punta Lara, Cabrera 731 (LP). Chaco, Schulz y Benitez 25 (SI). Corrientes: Saladas, Tabay, s. coll. 3692 (SI). Entre Ríos: Concordia, Schulz 134605 (LIL). Federación, Isthilart, Schulz 559 (SI). Formosa: Laishi, ruta prov. 5 entre Laishi y Tatané, Guaglianone et al. 716 (SI). Salta: San Ramón de la Nueva Orán, Río Piedras y Urundel, Vervoorst et al. 4649 (SI). Tucumán: Capital, Río Lules a San Felipe, Schreider 780 (SI).

BRASIL: Rio Grande do Sul, Arroyo Chanota, Krapovickas \& Cristobal 34231 (SI). 


\section{Canna indica $\mathrm{L}$.}

(Fig. 1C, 2A-B, 4D, 6B)

Granos de polen esferoidales, de (40) 60 (63) $\mu \mathrm{m}$, omniaberturados, equinados.

Espinas cónicas, agudas, de $3.5 \times 3 \mu \mathrm{m}$; densidad 9-13 espinas/campo de $400 \mu \mathrm{m}^{2}$.

Exina gris, extendida hasta la base de las espinas, éstas más claras. Exina corrugadoreticulada a rugado-plegada. Superficie de las espinas lisa, granular en la base.

Ejemplares de referencia seleccionados

ARGENTINA: Buenos Aires: La Plata, Gonnet, cerca de Papini y Bordenave, Delucchi 2342 (LP). Ensenada, Punta Lara, Dawson 943 (LP). Chaco: sin localidad, Baggi 3166 (SI). Corrientes: Capital, Isla Meza, Rodrigo 837 (LP).Tucumán: Capital, Lillo 51618 (LIL). Río Sali, Meyer 8641 (LIL). Entre Capital y Muñecas, Meyer 1345592 (LIL). Cruz Alta, Ranchillos, Bailetti 51609 (LIL). Tafí, orilla de la vía de Tafí al Cadillal, Herrera 345 (LIL). Santa Fe: Obligado, Tacuarendí, Pire 740 (SI).

BRASIL: Santa Catarina: Xaxim, Smith $y$ Reitz 12497 (SI).

GUATEMALA: Alta Verapaz, Cubilquitz, von Turckheim 4018 (LIL).

PARAGUAY: Presidente Hayes: Colonia Mennonita, Misión Nueva Vida, Arenas 1561 (SI).

PERU: Loreto: Santa María, Allard 22453 (LIL).

\section{Canna paniculata Ruiz et Pav.}

(Fig. 1D, 2F, 5C, 6A)

Granos de polen esferoidales, de (50) 68 (75) $\mu \mathrm{m}$, omniaberturados, equinados. Espinas cónicas, romas, ocasionalmente truncadas en el ápice, de $2.7 \times 3.3 \mu \mathrm{m}$; densidad 7-12 espinas/ campo de $400 \mu \mathrm{m}^{2}$. Exina extendida hasta la base de las espinas, de tono gris; espinas de valor similar. Superficie de la exina lisa, con micro-gránulos. Superficie de las espinas lisa, con micro-gránulos.
Ejemplares de referencia seleccionados

ARGENTINA: Buenos Aires: Delta del Paraná, Arroyo Manzano de Medina, Bridarolli 2216 (LP). Misiones: San Javier, Acaragua (Alto Uruguay), Bertoni 2774, 3682 (ambos LIL). Matto Quemado, s/c. 3706 (LIL). General Manuel Belgrano, Bernardo de Irigoyen, Hunziker 925 (LIL). San Antonio, Bertoni 445 (LIL). San Pedro, Alegría km. 367, Bertoni 1035 (LIL). San Martín, Puerto Leoní, Crisci 101 (LP).

\section{Canna variegatifolia Ciciarelli}

(Fig. 1B, 2C-D, 4B, 6G D)

Granos de polen esferoidales, de (72) 80 (81) $\mu \mathrm{m}$, omniaberturados, equinados. Espinas cónicas, con ápice agudo, isodiamétricas, $1.5 \times 1.5 \mu \mathrm{m}$; densidad 8-14 espinas/campo de $400 \mu \mathrm{m}^{2}$. La exina llega hasta la mitad de la altura de la espinas. Exina gris, espinas grises. Superficie rugulado-perforada. Superficie de las espinas lisa. Pared y espinas de igual valor.

\section{Ejemplares de referencia seleccionados}

ARGENTINA: Buenos Aires: Partido de Tres de Febrero, El Palomar, Hurrell y Bazzano 5671 (LP). La Plata, City Bell, a orillas del arroyo Rodríguez, 483 casi 20, Ciciarelli 16 (LP). City Bell, Alvear, entre 16 y Sarmiento, Ciciarelli 12 (LP). City Bell, Plaza Belgrano, Ciciarelli 13 (LP). Santa Fe: cerca de La Capital, Ciciarelli 14 (LP).

\section{DISCUSIÓN Y CONCLUSIONES}

Nair (1960) consideró que la morfología del polen de Canna podría ser un rasgo diagnóstico en táxones cultivados, mientras que Segeren \& Maas (1971) juzgaron esos rasgos como no significativos en especies silvestres. Este estudio y algunos precedentes (Tanaka 2001, Ciciarelli 2007, Ciciarelli \& Rolleri 2008) indican que, pese a la aparente uniformidad, ciertos rasgos de la pared de los granos de polen son específicos. 

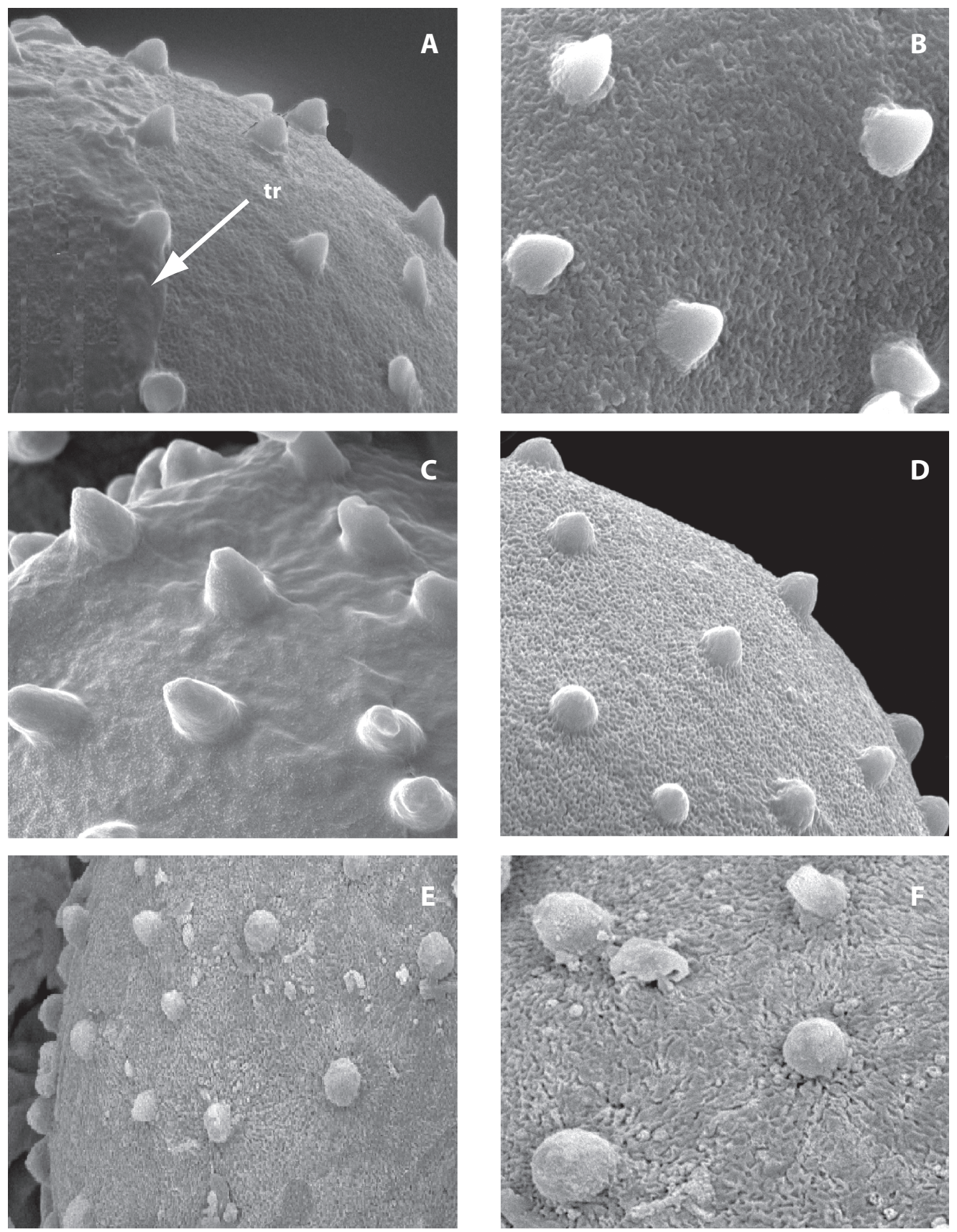

Fig. 5. Ornamentación de los granos de polen en especies de Canna. A-B: C. glauca. A: Superficie de la pared con pátina de trifina. B: Detalle de escultura de la pared y espinas. C: C. paniculata. D: C. fuchsina. E-F: C. compacta. E: Varias espinas, de forma irregular. F: Espinas digitiformes, pared estriada y microperforada. tr, trifina. Barra $=6 \mu \mathrm{m}$ en A, D, E; $4 \mu \mathrm{m}$ en $\mathrm{B}, \mathrm{F} ; 3 \mu \mathrm{m}$ en $\mathrm{C}$.

Fig. 5. Ornamentation of pollen grains in species of Canna. A-B: C. glauca. A: Tryphine patina over wall surface. B: Spines. C: C. paniculata. D: C. fuchsina. E-F: C. compacta. E: Several, irregular shaped spines. F: Digitiform spines, striated and microperforate wall. tr, tryphine. Bar $=6 \mu \mathrm{m}$ in $\mathrm{A}, \mathrm{D}, \mathrm{E} ; 4 \mu \mathrm{m}$ in $\mathrm{B}, \mathrm{F} ; 3 \mu \mathrm{m}$ in $\mathrm{C}$. 

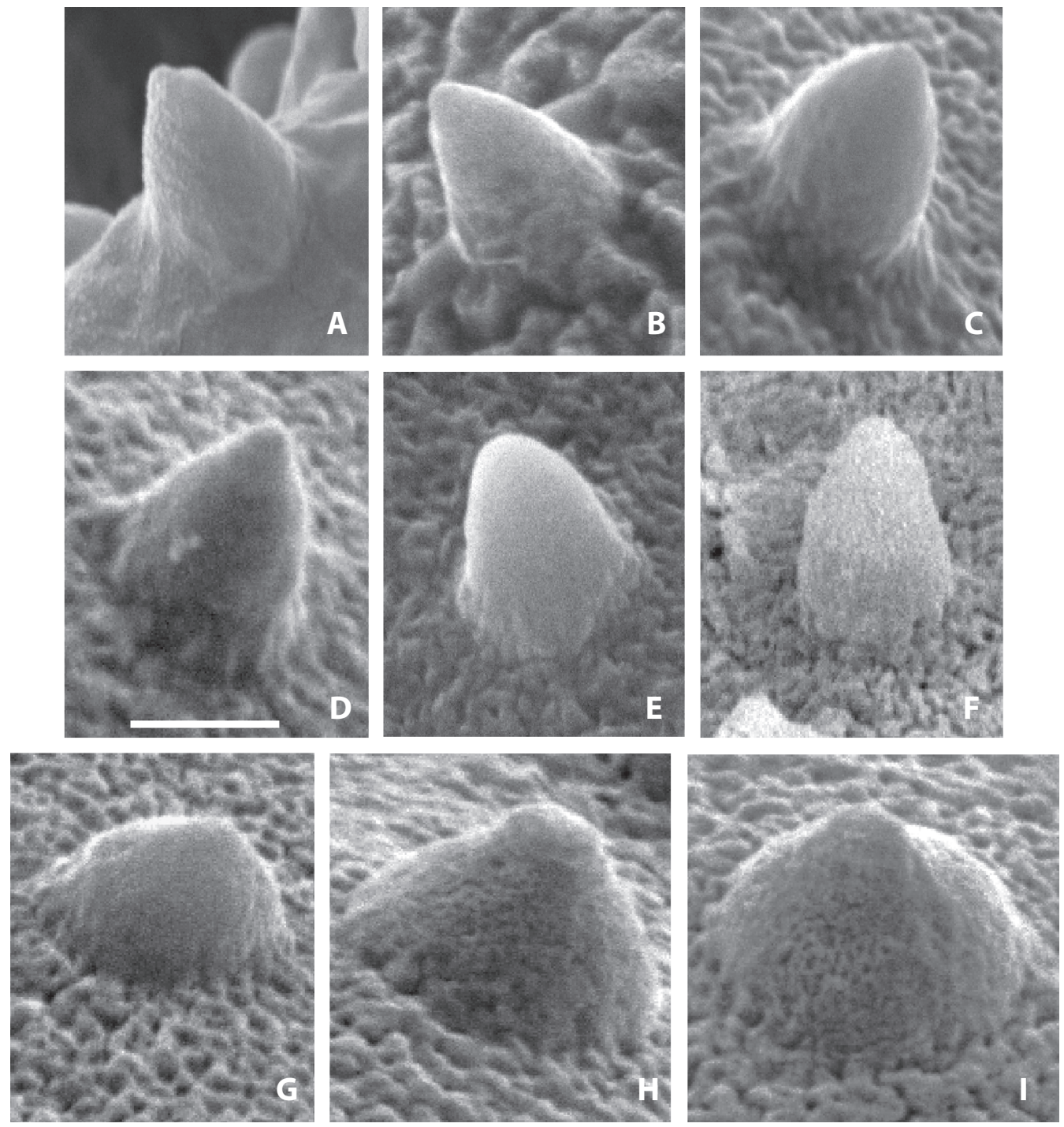

Fig. 6. Morfología de las espinas en granos de especies de Canna. A: C. paniculata. B: C. indica. C: C. ascendens. D: C. variegatifolia. E: C. glauca. F: C. compacta. G: C. fuchsina. H-I: C.coccinea. Barra $=4 \mu \mathrm{m}$ en A-B, D; $1,3 \mu \mathrm{m}$ en C, F; 2 $\mu \mathrm{m}$ en $\mathrm{E} ; 1,5$ en $\mathrm{G} ; 1,4 \mu \mathrm{m}$ en H-I.

Fig. 6. Morphology of the spines in pollen grain of species of Canna.. A: C. paniculata. B: C. indica. C: C. ascendens. D: C. variegatifolia. E: C. glauca. F: C. compacta. G: C. fuchsina. H-I: C.coccinea. Bar $=4 \mu \mathrm{m}$ in A-B, D; $1,3 \mu \mathrm{m}$ in C, F; 2 $\mu \mathrm{m}$ in $\mathrm{E} ; 1,5$ in $\mathrm{G} ; 1,4 \mu \mathrm{m}$ in H-I. 
La ornamentación equinada es un rasgo genérico en Canna, pero en los táxones estudiados, resultaron caracteres diagnósticos a nivel específico el tamaño de los granos, la superficie y valor de tonalidad de sus paredes, vistas con MEB, la forma y densidad de los procesos, sean éstos considerados espinas típicas o verrugas de aspecto espinuloso.

Los rasgos mencionados permiten obtener conclusiones en otros aspectos sistemáticos. Canna coccinea, considerada durante mucho tiempo sub C. indica (Segeren \& Maas 1971, Maas 1979, 1985, 1988, Maas \& Van de Kamer 2001 y Maas-Van de Kamer \& Maas 2003, 2008) fue reconocida como un táxon diferente por Ciciarelli \& Rolleri (2008) con base en rasgos vegetativos y florales. Este estudio demuestra que los caracteres del polen tales como el tamaño de los granos, la forma y densidad de los procesos de la ornamentación, la superficie de la exina y los patrones esculturales difieren significativamente en ambas especies y también permiten distinguirlas.

Canna fuchsina se estudió en colonias que crecen silvestres en la ribera rioplatense y áreas abiertas suburbanas de las provincias de Buenos Aires y Santa Fe, Argentina y se pudo constatar que las plantas forman granos de polen sin anormalidades que generan gametófitos masculinos también normales, con la producción consiguiente de frutos y semillas cuya germinación indica que se trata de una especie que se reproduce normalmente. La descripción y análisis de estos individuos son objeto de un trabajo aparte, pero dado que la morfología del polen ha sido un aspecto importante en el reconocimiento de este táxon, se la incluyó en este estudio; sus granos de polen sugieren afinidades con C. coccinea. Stanley (1987) citó numerosos ejemplares, fenotípicamente similares a C. fuchsina, también con flores de color rosa intenso y consideró que podrían ser parte del grupo diverso reunido sub $C$. generalis, que se naturalizan en Australia y crecen espontáneamente en localidades de Brisbane, Glandstone, Queensland y Perth. Aunque se han podido consultar fotografías muy claras de los materiales de herbario mencionados por
Stanley (1987) y se encontraron coincidencias exomorfológicas con las plantas de C. fuchsina de la Argentina, no se puede afirmar con seguridad que se trata del mismo táxon dado que no fue posible analizar los granos de polen. Las colonias halladas de C. fuchsina. podrían ser silvestres en origen o, lo que no es improbable, naturalizadas a partir de materiales introducidos como ornamentales, por vía de actividades humanas, tales como la jardinería, la floricultura o los movimientos de poblaciones humanas, accidentalmente o intencionadamente, algo que en Canna es un fenómeno común dado que sus flores e inflorescencias vistosas las hacen atractivas como plantas ornamentales.

Los estudios de esporodermo en Canna no son muy frecuentes. Wodehouse (1932) lo describió como constituido por una exina muy delgada y una intina transparente y muy gruesa y su estratificación fue considerada de difícil interpretación por Erdtman (1960 b) y Nair (1960). Pese a esas opiniones, Skvarla \& Rowley (1970) y Kress \& Stone (1982) coinciden en describir un esporodermo grueso, formado por una exina delgada, presente también en las espinas, y una gruesa intina celulósica, formada por dos capas, endintina y exintina.

De acuerdo con lo observado en los táxones estudiados, los granos de polen siempre son esferoidales, equinados y omniaberturados. La exina presenta diferentes tipos de superficie, desde lisas con algunos microgránulos dispersos o densos, como en los granos de C. paniculata hasta densamente corrugadas y microperforadas, como en los de $C$. indica. Esta capa es muy delgada, delicada y a menudo incompleta; se rompe con facilidad o presenta rajaduras que permiten ver la exintina, como sucede espontáneamente en granos de polen de C. indica.

La extensión de la exina ornamentada sobre las espinas es variable en los táxones estudiados. Este es un aspecto nuevo, no registrado previamente. Skvarla \& Rowley (1970), destacan la presencia no uniforme de esporopolenina entre las espinas de $C$. generalis y la presencia de trifina sobre espinas y exintina de ese táxon, se sugiere que existe alguna 
diferencia química entre la esporopolenina de la ectexina de la superficie, más delgada, y la que forma los procesos ornamentales, más compacta y resistente. Aquí se ha observado que la exina ornamentada se interrumpe en la base de las espinas, como en C. glauca, C. indica y C. paniculata. Además, se puede constatar su delgadez en granos rajados o en granos que se encuentran emitiendo tubos polínicos, como en $C$. fuchsina, $C$. indica y $C$. variegatifolia. En otros táxones esta capa llega a cubrir la mitad de la longitud de la espina, como en $C$. ascendens y C. variegatifolia, otro carácter que apoya la afinidad entre estas dos especies y en C. fuchsina. En C. coccinea y C. compacta la exina ornamentada no se interrumpe y cubre totalmente las espinas. La interrupción o continuidad de la exina en las espinas explicaría los diferentes valores de tono que se observan con MEB. La trifina superficial aparece en todos los táxones.

Las pruebas microquímicas efectuadas confirman lo observado. La exina, muy delgada y las espinas están constituidas por esporopolenina y se demostró que la trifina, sustancia que se origina en el tapete (Erdtman 1969) y se libera una vez que éste se desintegra (Skvarla \& Rowley 1970), aparece sobre la superficie de la exina en forma de pátinas irregulares, no siempre de manera uniforme ni completa, tapando ocasionalmente grupos amplios de espinas. También se confirmó la presencia de una gruesa intina celulósica.

\section{RESUMEN}

Se estudió la morfología de los granos de polen de ocho táxones de Canna, C. ascendens, C. coccinea, C. compacta, C. glauca, C. indica, C. paniculata, C. variegatifolia y C. fuchsina, nueva especie aún no descrita, que fueron estudiadas usando microscopio de luz y microscopio electrónico de barrido. Nosotros utilizamos la técnica de Wodehouse en muestras de 20 granos por espécimen para medir la intina con el microscopio de luz; y la densidad de espinas (en campos de $400 \mu \mathrm{m}^{2}$ ) con el microscopio electrónico de barrido. Los granos de polen son esféricos, equinados y omniaberturados. El esporodermo presenta una exina muy delgada cubriendo una intina gruesa. La superficie del esporodermo puede ser corrugada, microperforada, sub-reticulada, rugada, rugulada, plegada-estria- da, micro-estriada, microgranulada o lisa. Las espinas están formadas por exina, cubiertas total o parcialmente por trifina. La intina esta formada por dos capas, es la parte más gruesa de la pared. La ornamentación equinada es un rasgo genérico en Canna, pero el tamaño, la superficie y el color del polen, y la densidad y forma de las espinas, son rasgos diagnósticos de las especies. La morfología del polen apoya el tratamiento de $C$. coccinea y $C$. indica como especies diferentes. Canna fuchsina crece formando densas colonias silvestres en selvas ribereñas húmedas de las provincias de Buenos Aires y Santa Fe, Argentina; sus características sugieren relaciones no muy bien entendidas en el grupo de taxones, algunos son híbridos tales como $C$. $x$ generalis. Sin embargo, estas plantas muestran granos normales, bien formados, cercanos a los de C. coccinea, que germinan sobre el estigma de flores frescas, nosotros decidimos incluir su polen en este estudio.

Palabras clave: Canna,Cannaceae, polen, granos omniaberturados, ornamentación equinada, exina, espinas, intina, trifina, palinología.

\section{REFERENCIAS}

Bailey, L.H. 1923. Various cultigens and transfers in nomenclature. Gent. Herb. 1: 120.

Bouché, P.C. 1833. Mitheilung vieljähriger Beobachtungen uber die Gattung Canna. Linnaea 8: 141-168.

Bouché, P.C. 1844. Synoptische Zusammenstellung der Canna Arten, so wie zweier davon getrennten Gattungen. Linnaea 18: 483-495.

Ciciarelli, M.M. 1989. Las Cannaceae Link argentinas. Tesis Doctoral, Facultad de Ciencias Naturales y Museo de La Plata, Argentina.

Ciciarelli, M.M. 1995. Canna variegatifolia Ciciarelli sp. nov. (Cannaceae-Zingiberales). Rev. Museo La Plata, Bot. 14: 333-341.

Ciciarelli, M.M. 2007. Canna ascendens Ciciarelli (Cannaceae) una nueva especie de la provincia de Buenos Aires y comentarios de otras especies argentinas de este género. Darwiniana 45: 188-200.

Ciciarelli, M.M. \& C.H. Rolleri. 2008. Morfología, táxonomía y caracterización de siete especies neotropicales del género Canna (Cannaceae). Bot. Complutensis 32: 157-184.

Ciciarelli, M.M., C.H. Rolleri \& M.C. González. 2009. Canna fuchsina, una especie nueva para la ciencia, sus relaciones con otras especies silvestres del género y con el grupo C. $x$ generalis (Cannaceae-Zingiberales). Bot. Complutensis, in press. 
Chase, M.W., D.E. Soltis, R.G. Olmstead, D. Morgan, D.H. Les, B.D. Mishler, M.R. Duvall, R. Price, H.G. Hills, Y. Qui, A. Kron, J.H. Retting, E. Conti, J.D. Palmer, J.R. Manhart, K.J. Sytsma, H.J. Michaels, W.J. Kress, K.G. Karol, W.D. Clark, M. Hedren, B.S. Gaut, R.K. Jansen, K. Kim, C.F. Wimpee, J.F. Smith, G.R. Furnier, S.H. Strauss, Q. Xiang, G.M. Plunkett, P.S. Soltis, S.E. Swensen, S.E. Williams, P.A. Gadek, C.J. Quinn, L.E. Eguiarte, E. Golenberg, G.H. Learn; J. S. Graham, C.H. Barret, S. Dayanandan \& V.A. Albert. 1993. Phylogenetics of the seed plants: an analysis of nucleotide sequences from the plastid gene rbcL. Ann. Missouri Bot. Gard. 80: 528-580.

Dahlgren, R.M. \& F.N. Rassmusen. 1983. Monocotyledon evolution: characters and phylogenetic estimation. Evol. Biol. 16: 255-395.

Delin, W. \& W.J. Kress. 2000. Cannaceae, p. 378. In Flora. Z.Y. Wu, P.H. Raven \& D.Y. Hong (eds.). Flora of China. Vol. 24 (Amaryllidaceae to Zingiberaceae). Science, Beijing and Missouri Botanical Garden, San Luis, Misuri, EEUU.

Erdtman, G. 1960a. The acetolysis method, a revised description. Svensk Bot.Tidskr. 54: 561-564.

Erdtman, G. 1960b. Pollen walls and angiosperm phylogeny. Bot. Not. 113: 41-45.

Erdtman, G. 1969. Handbook of palinology. An introduction to the study of pollen grains and spores. Munksgaard, Copenague, Dinamarca.

Horaninow, P.F. 1862. Prodromus Monographie Scitaminearum. San Petersburgo, Rusia.

Judd, W.S., C.S. Campbell, E.A. Kellog, P.F. Stevens \& M.J. Donoghue. 2002. Plant Systematics. A phylogenetic approach. Sinauer, Sunderland, Massachusetts, EEUU.

Kranzlin, F. 1912. Cannaceae, p. 1-77. In A. Engler (ed.). Das Pflanzenreich, IV. Wilhelm Engelmann, Leipzig, Alemania.

Kress, W.J. \& D.E. Stone. 1982. Nature of the sporoderm in monocotyledons, with special reference to the pollen grains in Canna and Heliconia. Grana 21: 129-148.

Kress, W.J. 1990. The phylogeny and classification of the Zingiberales. Ann. Missouri Bot. Gard. 77: 698-721.

Kress, W.J. 1995. Phylogeny of the Zingiberanae: morphology and molecules, p. 443-460. In P.J. Rudall, P.J. Cribb, D.F. Cutler \& C.J. Humphries (eds.). Monocotyledons: Systematics and evolution. Royal Botanic Gardens, Kew, Londres.
Kress, W.J. \& L. Prince. 2000. Cannaceae, p. 310-314. In Flora of North America Editorial Committee, Flora of North America and México. Oxford University, Nueva York, EEUU.

Kress, W.J., L.M. Prince, W.J. \& E.A. Zimmer. 2001. Unraveling the evolutionary radiation of the families of the Zingiberales using mophological and molecular evidence. Syst. Biol. 50: 926-944.

Maas, P.J.M. 1979. Cannaceae. In R. A. Howard (ed.). Flora of the Lesser Antilles 3. Harvard University, Massachusetts, EEUU.

Maas, P.J.M. 1985. Cannaceae. In A. R. A. Gorts-van-Rijn (ed.). Flora of the Guianas. Koeltz Scientific Books, Alemania.

Maas, P.J.M. \& H. Maas. 1988. Cannaceae, p.1-9. In G. Harling and L. Andersson (eds.). Flora of Ecuador. Gotemburgo y Estocolmo, Suecia.

Maas, P.J.M. \& H. Maas-Van de Kamer. 2001. Cannaceae. In W.D. Stevens, C. Ulloa, A. Pool \& O. M. Montiel (eds.). Flora of Nicaragua. Monographs of Systematic Botany, Missouri Botanical Garden 85: 565-66.

Maas-Van de Kamer, H. \& P.J.M. Maas. 2003. Cannaceae. In B.E. Hammel, M. Grayum, H.C. Herrera and E. Zamor (eds.). Manual de Plantas de Costa Rica. Monographs of Systematc Botany. Missouri Bot. Gard. 92: 384-385.

Maas-Van de Kamer, H. \& P.J.M. Maas. 2008. The Cannaceae of the World. Blumea 53: 247-318.

Nair, P.K.K. 1960. Pollen grains of cultivated plants. I: Canna. J. Indian Bot. Soc. 39: 373-381.

Punt, W., P.P. Hoen, S. Backmore, S. Nilsson \& A. Le Thomas. 2007. Glossary of pollen and spore terminology. Rev. Palaeobot. Palynol. 143: 1-81.

Segeren, W. \& P.J.M. Maas. 1971. The genus Canna in Northern South America. Acta Bot. Netherl. 20: 663-680.

Skvarla, J.J. \& J.R. Rowley. 1970. The pollen wall of Canna and its similarity to the germinal apertures of other pollen. American J. Bot. 57: 519-529.

Skvarla, J.J. \& J.R. Rowley. 1974. Origin of the inner intine in pollen of Canna. $32^{\text {nd }}$ Annals and proceedings Electron Microscopy Society of America, St. Louis, Misuri, EEUU.

Skvarla, J.J. \& J.R. Rowley. 1975. The glycocalyx and initiation of exine spinules on microspores of Canna. American J. Bot. 62: 479-485. 
Smith, J.F., W.J. Kress \& E.A. Zimmer. 1993. Phylogenetic analysis of the Zingiberales based on $\mathrm{rbcL}$ sequences. Ann. Missouri Bot. Gard. 80: 50-66.

Stanley, T.D. 1987. Cannaceae. In Flora of Australia. Vol. 45. Australian Gov. Camberra, Australia.

Stevenson, D.W. \& H. Loconte. 1995. Cladistic analysis of the monocot families. In P. J. Rudall, P.J. Cribb, D.F. Cutler \& C. J. Humphries (eds.). Monocotyledons: Systematics and evolution. Royal Botanic Gardens, Kew, Londres.

Tanaka, N. 2001. Taxonomic revision of the family Cannaceae in the New World and Asia. Makinoa, new series 1: 1-74.

Tomlinson, P.B. 1962. Phylogeny of the Scitaminae: Morphological and anatomical considerations. Evolution 16: $192-213$.
Tomlinson, P.B. 1969. Commelinales-Zingiberales. In C.R. Metcalfe (ed.). Anatomy of the Monocotyledons. Vol. 3. Clarendon, Oxford, Londres.

Tutin, T.G., V.H. Heywood, N.A. Burges, D.M. Moore, D.H. Valentine, S.M. Walters \& D.A. Webb. (eds.). 1980. Flora Europaea 5. Alismataceae to Orchidaceae (Monocotyledons). Cambridge University, Cambridge, Inglaterra.

Winkler, H. 1930. Musaceae, Cannaceae. In A. Engler \& K. Prantl (eds.). Die naturlichen Pflanzenfamilien. Vol. 15a. Wilhelm Engelmann, Leipzig, Alemania.

Wodehouse, R.P. 1932. Tertiary pollen. I. Polen of the living representatives of the Green River flora. Bull. Torrey Bot. Club 59: 313-340.

Wodehouse, R.P. 1935. Pollen grains. Their structure, identification and significance in science and medicine. Mc. Graw Hill, Nueva York, EEUU. 
